\title{
ON THE INNER RADIUS OF UNIVALENCY FOR NON-CIRCULAR DOMAINS
}

\author{
MATTI LEHTINEN
}

Let $A$ be a domain in the extended complex plane, conformally equivalent to a disc. We denote by $\varrho_{A}|d z|$ the Poincaré metric in $A$, a conformal invariant so normalized that the density $\varrho_{A}$ satisfies $\varrho_{H}(z)=(2 \operatorname{Im} z)^{-1}$ for the upper half-plane $H$. The norm of the Schwarzian derivative $S_{f}$ of a locally injective meromorphic function $f$ in $A$ is the number

$$
\left\|S_{f}\right\|_{A}=\sup _{z \in A}\left|S_{f}(z)\right| / \varrho_{A}(z)^{2} .
$$

The inner radius of univalency $\sigma(A)$ of $A$, introduced by Lehto ([3], [4]), is the supremum - or maximum - of numbers $a$ such that $\left\|S_{f}\right\|_{A} \leqq a$ is a sufficient condition for $f$ to be univalent in $A$. A Möbius transformation of a domain does not change its inner radius of univalency. By a result of Gehring [1], $\sigma(A)$ is positive only if $A$ is bounded by a quasicircle. Also, if $\left\|S_{f}\right\|_{A}<\sigma(A)$, then $f(A)$ is bounded by a quasicircle, and hence is a Jordan domain.

The classical results of Nehari [5] and Hille [2] show that if $A$ is a disc, then $\sigma(A)=2$. If the monotonicity of $\varrho_{A}(z)$ with respect to $A$ is taken into account, it is not difficult to conclude that $\sigma(A) \leqq 2$ for any $A$. Our aim is to sharpen this inequality by proving

Theorem. For any domain $A$, not Möbius equivalent to a disc, $\sigma(A)<2$.

The proof of the Theorem rests on two lemmas, one analytical and the other geometrical:

Lemma 1. Let $B_{k}=\{z \mid 0<\arg z<k \pi\}, 1<k<2$. Then

$$
\sigma\left(B_{k}\right) \leqq 4 k-2 k^{2} .
$$

Proof. We shall establish the existence of a conformal map $f: B_{k} \rightarrow E$ such that $\left\|S_{f}\right\|_{B_{k}}=4 k-2 k^{2}$ and $E$ is not a Jordan domain. In fact, set

$$
E=\{z|| \arg z \mid<k \pi / 2\} \cap\{z|| \arg (1-z) \mid<k \pi / 2\} .
$$


The Schwarzian derivative of a conformal map $g: H \rightarrow E \cap H$ with $g(0)=1, g(\infty)=0$, $g(1)=\infty$ is given by (see e.g. [6, p. 203])

$$
S_{g}(z)=\frac{4-k^{2}}{2 z^{2}}+\frac{2 k-k^{2}}{2(z-1)^{2}}+\frac{k^{2}-2 k}{2 z(z-1)} .
$$

A conformal map $h: H \rightarrow E$ is obtained if the map $z \mapsto g\left(z^{2}\right)$, defined in the first quadrant of the plane, is reflected over the imaginary axis. The composition rule for the Schwarzian yields

$$
S_{h}(z)=\frac{1-k^{2}}{2 z^{2}}+\frac{4 k-2 k^{2}}{\left(z^{2}-1\right)^{2}}
$$

Now $S_{\varphi}(z)=\left(1-k^{2}\right) /(2 z)^{2}$ for $\varphi(z)=z^{k}$. Set $f=h \circ \varphi^{-1}$. Then, by $\left\|S_{f}\right\|_{B_{k}}=$ $\left\|S_{h}-S_{\varphi}\right\|_{H}$ and an elementary computation,

$$
\left\|S_{f}\right\|_{B_{k}}=\sup _{y \neq 0} \frac{4 y^{2}\left(4 k-2 k^{2}\right)}{\left(x^{2}-y^{2}-1\right)^{2}+4 x^{2} y^{2}}=4 k-2 k^{2} .
$$

Remark. Lemma 1 complements a result by Lehto [4], who proved an inequality opposite to (1). We have thus actually established the equality

for $1 \leqq k \leqq 2$.

$$
\sigma\left(B_{k}\right)=4 k-2 k^{2}
$$

Lemma 2. Assume $A \subset H$ is a Jordan domain having two finite boundary points $a$ and $b$ on the real axis such that the open interval $(a, b)$ is in the complement of $\partial A$. Then $A$ lies in the opening of an obtuse angle whose both sides contain a point of $\partial A$ at an equal distance from the vertex.

Proof. Choose an $s, 0<s<(b-a) / 4$. A positive $r$ exists such that the rectangle $R$ with vertices $a+s, b-s, b-s+i r, a+s+i r$ is in the complement of $A$. For each point $p$ in $\bar{R} \cap H$ one may consider the smallest obtuse angle $B(p)$ with vertex $p$ containing $A$ in its opening. Let $q_{1}(p)$ and $q_{2}(p)$ be the points of $\partial A$ closest to $p$ on the left and right side of $B(p)$, respectively. Set

$$
\psi(p)=\frac{\left|p-q_{1}(p)\right|}{\left|p-q_{2}(p)\right|} .
$$

For any fixed point $a+s+i t, 0<t<r$, let $p$ move on the right side of $B(a+s+i t)$ from $a+s+i t$ towards $q_{2}(a+s+i t)$. Evidently $\psi(p)$ then increases monotonically from 0 to values $>1$. There is a unique $p=q_{0}(t)$ at which either $\psi(p)=1$ or $\psi$ has a finite jump from a value $<1$ to a value $>1$. Now consider $q_{1}\left(q_{0}(t)\right)$. Its real part is a monotonically increasing function of $t$, and a jump in $\psi(p)$ at $q_{0}(t)$ means a jump in $\operatorname{Re} q_{1}\left(q_{0}(t)\right)$. Since the latter ranges between $a$ and $a+s$, there can be only a denumerab!e number of $t$ 's associated with a jump, and a $t_{0}$ can be chosen such that $B\left(q_{0}\left(t_{0}\right)\right)$ is the desired angle. 
The proof of the Theorem is now evident: Any Jordan domain $A$ not a disc is Möbius equivalent to a domain $A^{\prime}$ of the type described in Lemma 2, and hence also to a domain $A^{\prime \prime}$ in some $B_{k}, 1<k<2$, with $\left\{1, e^{i k \pi}\right\} \subset \partial A^{\prime \prime}$. The restriction to $A^{\prime \prime}$ of the map $f$, discussed in the proof of Lemma 1 , carries $A^{\prime \prime}$ onto a domain not bounded by a Jordan curve, and the monotonicity of the density of the Poincaré metric implies

$$
\left\|S_{f \mid A^{\prime \prime}}\right\|_{A^{\prime \prime}} \leqq\left\|S_{f}\right\|_{B_{k}}=4 k-2 k^{2}<2 .
$$

\section{References}

[1] Gehring, F. W.: Univalent functions and the Schwarzian derivative. - Comment. Math. Helv. $52,1977,561-572$.

[2] Hille, E.: Remarks on a paper by Zeev Nehari. - Bull. Amer. Math. Soc. 55, 1949, 552-553.

[3] LeHto, O.: Domain constants associated with Schwarzian derivative. - Comment. Math. Helv. $52,1977,603-610$.

[4] Lehto, O.: Remarks on Nehari's theorem about the Schwarzian derivative and schlicht functions. - To appear.

[5] Nehari, Z.: The Schwarzian derivative and schlicht functions. - Bull. Amer. Math. Soc. 55, $1949,545-551$.

[6] Nehari, Z.: Conformal mapping. - McGraw-Hill Book Company, Inc., New York-TorontoLondon, 1952.

University of Helsinki

Department of Mathematics

SF-00100 Helsinki 10

Finland

Received 29 August 1979 